\title{
基于混沌自适应粒子群人工神经网络的气体在聚合物中的溶解模型
}

\author{
李孟山 ${ }^{a, b} \quad$ 黄兴元 $^{*, a} \quad$ 柳和生 $^{a} \quad$ 柳炳祥 $^{b}$ 武燕 ${ }^{b}$ 艾凡荣 ${ }^{a}$ \\ ( ${ }^{a}$ 南昌大学 机电工程学院 南昌 330031) \\ $\left(^{b}\right.$ 景德镇陶瓷学院 信息工程学院 景德镇 333001)
}

\begin{abstract}
摘要 为提高溶解预测模型的效率和关联度, 建立基于混沌理论、自适应粒子群优化(PSO)算法和反向传播(BP)算法的 混沌自适应 PSO-BP 神经网络模型, 并对二氧化碳 $\left(\mathrm{CO}_{2}\right)$ 在聚苯乙烯 $(\mathrm{PS})$ 和聚丙烯 $(\mathrm{PP})$ 中、氮气 $\left(\mathrm{N}_{2}\right)$ 在 PS 中的溶解度进 行预测试验. 模型选用压力和温度作为输入参数, 使用试探法确定隐含层结点个数为 8 , 输出为预测的溶解度. 模型融 合混沌理论、自适应 PSO 和 BP 算法各自的优势，提高了训练速度和预测精度. 结果表明，混沌自适应 PSO-BP 神经网 络有很好的预测能力, 预测值与实验值相当吻合, 通过与传统 BP 神经网络和 PSO-BP 神经网络的比较可知, 其预测精 度和相关性均明显较优, 预测平均绝对误差(AAD), 标准偏差(SD)和平方相关系数 $\left(R^{2}\right)$ 分别为 $0.0058,0.0198$ 和 0.9914. 关键词＼cjkstart溶解预测; 聚合物; 神经网络; 粒子群; 混沌理论
\end{abstract}

\section{Solubility Prediction of Gases in Polymers based on Chaotic Self-adaptive Particle Swarm Optimization Artificial Neural Networks}

\author{
Li, Mengshan ${ }^{a, b} \quad$ Huang, Xingyuan ${ }^{*, a} \quad$ Liu, Hesheng $^{a} \quad$ Liu, Bingxiang ${ }^{b}$ \\ $\mathrm{Wu}, \mathrm{Yan}^{b} \quad \mathrm{Ai}$, Fanrong $^{a}$ \\ ( ${ }^{a}$ Nanchang University, College of Mechanical and Electric Engineering, Nanchang 330031) \\ ( ${ }^{b}$ JingDeZhen Ceramic Institute, School of Information Engineering, JingDeZhen 333001)
}

\begin{abstract}
Solubility is one of the most important physicochemical properties of polymer compounds, which determines the compatibility of blending system. To enhance the performance of artificial neural networks (ANN) and improve the efficiency and correlation of prediction of gas solubility in polymers, in this work, a novel ANN model based on chaos theory, self-adaptive particle swarm optimization (PSO) algorithm and back propagation (BP) algorithm is proposed, hereafter called CSAPSO-BP ANN. In the CSAPSO-BP ANN, the conventional PSO algorithm is modified by using chaos theory and self-adaptive inertia weight factor to overcome its premature convergence problem. Then the CSAPSO-BP ANN trained by hybrid algorithm which combined the modified PSO and BP algorithm has been employed to investigate carbon dioxide $\left(\mathrm{CO}_{2}\right)$ solubility in polystyrene (PS), polypropylene (PP) and nitrogen $\left(\mathrm{N}_{2}\right)$ solubility in PS, respectively. The CSAPSO-BP ANN model which consisted of three layers with one hidden layer, two input nodes including temperature and pressure, 8 hidden nodes which obtained by heuristics and one output node that is the solubility of gases in polymers was designed. The model combined the abilities of chaos theory, PSO algorithm and BP algorithm, accelerated the training speed of ANN and improved the prediction accuracy. Results obtained in this work indicate that the CSAPSO-BP ANN is an effective method for prediction of gas solubility in polymers in a wide range of pressure and temperature. The comparison between different neural networks was carried out in detail to reveal the proposed CSAPSO-BP ANN outperforms the traditional BP NN and PSO-BP NN. The values of average absolute deviation (AAD), standard deviation (SD) and squared correlation coefficient $\left(R^{2}\right)$ are $0.0058,0.0198$ and 0.9914 , respectively. The statistical data demonstrate that the CSAPSO-BP ANN model is a faster, more reliable and accurate method, and has an excellent prediction capability with high-accuracy and has a good correlation between prediction values and experimental data.
\end{abstract}

Keywords solubility prediction; polymer; neural network; particle swarm optimization; chaos theory

\section{1 引言}

溶解性是衡量熔体兼容性的重要物理化学属性之 一, 在材料加工过程中, 熔体溶解性研究一直为材料学 科的研究热点, 特别是气体在聚合物中的溶解性研究具
有极大的应用前景，它对微孔发泡等泡孔材料领域起着 决定性作用，可给材料加工工序的最优化设计提供重要 依据 ${ }^{[1]}$, 近年来, 研究者们对此领域的研究越来越关 注 $^{[2 \sim 4]}$. 目前, 溶解性主要有实验和预测两种研究方法, 实验方法主要有相分离法、压力法、重力法、体积法和

* E-mail: huangxingyuan001@126.com

Received February 15, 2013; published March 15, 2013.

Project supported by the National Natural Science Foundation of China (No. 20664002) and Graduate Student Innovation Fund by Nanchang University (No. cx2012011).

项目受国家自然科学基金(No. 20664002)和南昌大学研究生创新专项资金(No. cx2012011)资助. 
色层法等 ${ }^{[5 \sim 7]}$, 预测方法主要分有限元法、状态方程法 和计算机模型法等 ${ }^{[8,9]}$. 但实验方法耗时、费力, 经济开 销也大，同时也容易受实验条件等因素的制约，从而影 响实验效果. 在传统预测模型中, 由于各种原因使预测 精度与适应性不高, 与实验值偏差也较大而未达到满意 的预测目的. 为了解决溶解性数据相对缺乏的问题, 研 究可行、高效且精度高的预测模型将是未来研究热点之 一.

从气体在聚合物中的溶解性来看, 影响溶解性的因 素很多, 如温度、压力和分子的极性等 ${ }^{[2]}$, 这些因素与溶 解行为之间在很大程度上表现出非线性关系, 特别是在 高温高压条件下表现出的非线性特征使传统的研究方 法相对吃力. 近年来, 人工神经网络(ANN)技术逐渐被 应用到化学工程领域 ${ }^{[10 ~ 12]}$, 神经网络自学习、自组织、 容错以及模拟复杂的非线性关系的能力使其特别适合 解决上述类似的非线性问题. 目前, 研究者也提出了一 些基于神经网络的溶解度预测模型, 如 Bakhbakhi ${ }^{[13]}$ 和 Lashkarbolooki ${ }^{[14]}$ 等通过比较神经网络和几种状态方程 的溶解度预测方法, 得到神经网络方法比状态方程预测 精度更高的结论. 然而, 神经网络预测的可靠性与精确 性在很大程度上依赖于网络的训练算法, 其中反向传播 (Back Propagation BP)最为常用, 如 Eslamimanesh ${ }^{[15]}$ 和 Gharagheizi ${ }^{[10]}$ 等提出基于梯度下降 $\mathrm{BP}$ 神经网络的预测 模型, 虽然得到了较好的预测效果, 但 BP 算法有容易 陷入局部最优、依赖于初始权值的选择等缺陷, 使其收 玫速度和逼近程度都很不理想. 然而, 神经网络良好性 能的实现却相当依赖于神经网络的充分训练, 因此, 训 练算法对于神经网络的性能有着决定性影响. 研究发 现, 神经网络的训练可归属于网络结构和参数调整的最 优化问题. 近年来, 随着学科间的相互交叉、相互渗透 和相互促进, 研究者开始将各类智能优化算法应用于网 络训练, 因此, 神经网络与各种智能优化算法有机结合 也成为必然的研究热点, 如与遗传算法(Genetic Algorithm GA)、模拟退火算法(Simulated Annealing SA)、粒 子群优化(Particle Swarm Optimization PSO)算法、模糊 理论、小波分析理论和混沌理论(chaos theory) 等相结 合 ${ }^{[16,17]}$, 即形成具有柔性信息处理能力的 “混合神经网 络”.

粒子群算法是一种群体智能进化算法, 与遗传算法 和模拟退火算法相比, 粒子群算法相对简单, 容易实现 且参数调整少, 是一种全局最优搜索算法, 具有很好的 应用前景. 研究者 $A h m a d i^{[18]}$ 等将粒子群优化(PSO)算法 应于神经网络训练, 得到了一种高效的预测模型. 单一 的 PSO 算法虽然有较强的全局最优搜索能力, 但在全 局最优值附近, 搜索过程变慢, 又可能会陷入局部最优, 此时粒子在最优值附近, 属于局部解问题, 而 BP 算法 却正好有很强的局部搜索能力, 若将 PSO 和 BP 算法相 互融合在一起, 可相互促进和相互补充. Zhang 等 ${ }^{[19]}$ 研
究者提出将 PSO 和 BP 算法结合形成的混合算法应用于 前馈神经网络的训练, 结果表明混合算法比单一的 PSO 或 BP 算法在训练性能和效率上明显优越. 虽然 Zhang 等 ${ }^{[19]}$ 研究者实现了 PSO 和 BP 算法的结合, 但只结合两 种算法优势而提高训练能力, 没有从根本上解决局部最 优问题. 为此, 本文试想将 PSO 算法较强的全局最优搜 索能力和 BP 算法快速的局部搜索能力相结合的同时, 考虑引入权值自适应策略和混沌理论，对 PSO 算法进 行性能改进，提高收敛速度和精度，并避免算法陷入局 部最优, 实现网络充分训练. 由于混沌理论系统具有随 机性、遍历性和规律性等优点, 能在训练寻优过程中改 进传统粒子群算法中粒子的更新策略并避免 PSO 算法 的早熟问题, 提出混沌自适应 PSO-BP 神经网络模型. 最后，应用提出的混沌自适应 PSO-BP 神经网络模型对 气体在聚合物中的溶解性进行预测, 再通过与传统 BP 神经网络和 PSO-BP 神经网络的比较来评价混沌自适应 PSO-BP 神经网络的性能.

\section{2 模型理论}

与遗传算法和模拟退火算法等群体智能算法相比, 粒子群优化(PSO)算法容易实现, 且需要调整的参数少, 收敛速度和效率均较优，成为当今群体智能算法的典 型. 粒子群算法由 $m$ 个粒子组成, 每一个粒子都想象成 $n$ 维搜索空间中的潜在解, 在求解迭代过程中粒子通过 公式(1)和(2)来更新自己的速度和位置 ${ }^{[18]}$.

$$
\begin{gathered}
v_{i, d}^{k+1}=\omega v_{i, d}^{k}+c_{1} \operatorname{rand}()\left(p_{i, d}^{k}-x_{i, d}^{k}\right)+c_{2} \operatorname{rand}()\left(p_{g, d}^{k}-x_{i, d}^{k}\right) \\
x_{i, d}^{k+1}=x_{i, d}^{k}+v_{i, d}^{k+1}
\end{gathered}
$$

式中, $i=1, \ldots, m ; \omega$ 为惯性权重因子, $x_{i, d}^{k}$ 和 $v_{i, d}^{k}$ 分别 为粒子 $i$ 在第 $k$ 次迭代中第 $d$ 维的速度和位置; $c_{1}$ 和 $c_{2}$ 为学习因子; $p_{i, d}^{k}$ 为粒子 $i$ 在第 $d$ 维空间中个体极值位 置; $p_{g, d}^{k}$ 为群体在第 $d$ 维空间中的全局极值位置.

在混沌自适应 PSO-BP 神经网络模型中, 惯性权重 因子的调整采用自适应调整策略，惯性权重因子 $\omega$ 通 过以下公式调整:

$$
\omega=\omega_{\max }-\operatorname{Pgbest}(k) / \text { Plbest }_{\mathrm{ave}}-\left(\omega_{\max }-\omega_{\min }\right) \times k / k_{\max }
$$

其中, $\omega_{\max }$ 和 $\omega_{\min }$ 为权值最大值和最小值; $\operatorname{Pgbest}(k)$ 为第 $k$ 次迭代时的全局最优解; Plbest $\mathrm{ave}_{\text {av }}$ 为部最好适应值的 平均值; $k_{\max }$ 为最大迭代次数.

混沌为具有随机性的运动状态, 由混沌变量来呈现 混沌状态, 也即混沌变量具有随机性、遍历性和规律性 特点. 利用变量的这些混沌特征和经典的 Logistic 方程 来实现混沌变量的演变并进行优化搜索, 能促进算法跳 出局部最优. 粒子按如下步骤产生混沌点列 ${ }^{[20]}$.

将粒子当前 $x_{i}$ 每一维的位置 $x_{i, d}$ 按式(3)映射到 [0, 1]区间上: 


$$
c x_{i, d}=\frac{x_{i, d}-a_{d}}{b_{d}-a_{d}}
$$

其中, $\left[a_{d}, b_{d}\right]$ 为 $d$ 维变量 $x_{i, d}$ 的定义域.

按式(4)迭代 $K$ 次产生的混沌序列 $c x_{i, d}^{1}, c x_{i, d}^{2}, \ldots, c x_{i, d}^{K}$.

$$
c x_{\mathrm{i}}^{k+1}=4 c x_{i}^{k}\left(1-c x_{i}^{k}\right), k=1,2, \cdots, K
$$

其中, $c x_{i}$ 为混沌变量; $c x_{i}^{k}$ 为 $c x_{i}$ 在 $k$ 次迭代后的值; $K$ 为混沌映射迭代次数.

混沌自适应 PSO-BP 神经网络以自适应 PSO 算法训 练神经网络为主导, 模型将自适应 PSO 算法、BP 算法 和混沌理论相互融合, 发挥各自的优势. 首先对网络进 行初始化, 采用自适应 PSO 算法对神经网络进行训练, 评价各粒子的初始适应度值, 产生混沌点列得到粒子的 新位置, 再评价新粒子的适应值, 若在训练进化过程中 适应度函数值无任何改变, 说明满足局部搜索条件, 则 执行 BP 算法进行局部快速搜索; 若从局部搜索得到的 适应度更好, 则将局部最优更新为 PSO 算法的全局最 优, 重复此过程直到结束条件满足 ${ }^{[19]}$, 此时网络的全局 最优即为网络的输出.

\section{3 预测实验}

\section{1 实验数据}

研究的聚合物有聚苯乙烯(PS)和聚丙烯(PP), 气体 有二氧化碳 $\left(\mathrm{CO}_{2}\right)$ 和氮气 $\left(\mathrm{N}_{2}\right)$. 经篮选和精简获得的实 验数据, 建立一个包含 283 组数据的实验数据库, 如表 1 统计所示. 根据聚合物和气体的种类, 将实验数据库 分成 $\mathrm{PS} / \mathrm{CO}_{2} 、 \mathrm{PS} / \mathrm{N}_{2}$ 和 $\mathrm{PP} / \mathrm{CO}_{2}$ 三个子库, 同时将每个子 库随机分成训练集、验证集和测试集三个子集, 其中训 练集用于训练并产生网络; 验证集用于验证网络的可靠 性; 测试集用于溶解预测. 为了提高网络的泛化能力,
将数据库数据约 $70 \%$ (即 199 组)用于网络训练, 验证和 测试分别为 45 和 39 组.

模型采用计算平均绝对误差(Average Absolute Deviation, $\mathrm{AAD}$ )、标准偏差(Standard Deviation, $\mathrm{SD}$ )和平方 相关系数(Squared Correlation Coefficient, $R^{2}$ )进行精度 和可靠性评价. 其中 $\mathrm{AAD}$ 和 $\mathrm{SD}$ 定义为:

$$
\begin{aligned}
& A A D=\frac{1}{N} \sum_{i=1}^{N} \frac{|\operatorname{Pre}(i)-\operatorname{Exp}(i)|}{\operatorname{Exp}(i)} \\
& S D=\sqrt{\frac{1}{N} \sum_{i=1}^{N}\left(x_{i}-x\right)^{2}}
\end{aligned}
$$

其中, $N$ 为样本个数, $\operatorname{Pre}(i)$ 为网络模型预测值, $\operatorname{Exp}(i)$ 为 实验实际值, $x$ 是 $N$ 个样本数据 $x_{i}$ 的平均值.

\section{2 实验模型}

人工神经网络的健壮性、收玫速度和预测精度, 一 方面依赖其训练算法, 另一方面依赖网络的结构, 网络 结构主要包括网络层数和各层的神经元结点个数. 研究 表明, 一个包含 3 层的神经网络几乎能模拟所有线性和 非线性问题，因此网络结构设计的重点是各层的结点个 数, 输入层与输出层结点个数主要由实际问题的影响因 素直接给出, 而隐层结点个数一般采用经验公式法和试 探法确定 ${ }^{[15,19]}$.

本文采用 3 层神经网络结构, 根据特定气体和聚合 物的溶解问题, 主要是分析溶解压力 $P$ 和温度 $T$ 对溶解 度的影响, 所以在输入层选择压力 $P$ 和温度 $T$ 作为输入 参数, 即输入结点个数为 2 ; 输出层中溶解度作为输出, 结点个数为 1 . 本文采用试探法优化得出隐层中神经元 个数, 个数从 3 增至 15 , 共得到 13 个混沌自适应 PSO-BP 神经网络模型, 通过分别计算各模型所得的 $A A D, S D, R^{2}$ 和最好适应值来确定隐层结点的最佳个数,

\begin{tabular}{|c|c|c|c|c|c|c|}
\hline 聚合物 & 气体 & 温度/K & 压力 $/ \mathrm{MPa}$ & 溶解度 $(\mathrm{g} / \mathrm{g})$ & 数据组数 & 参考文献 \\
\hline \multirow{5}{*}{ PS } & \multirow{5}{*}{$\mathrm{CO}_{2}$} & $338.22 \sim 362.50$ & $3.710 \sim 24.650$ & $0.03714 \sim 0.19110$ & 15 & [21] \\
\hline & & $372.20 \sim 383.22$ & $2.068 \sim 42.810$ & $0.01231 \sim 0.16056$ & 34 & {$[21 \sim 24]$} \\
\hline & & $402.51 \sim 413.20$ & $5.140 \sim 20.036$ & $0.01462 \sim 0.20190$ & 16 & {$[21,22]$} \\
\hline & & $423.15 \sim 473.15$ & $2.159 \sim 20.151$ & $0.00688 \sim 0.06870$ & 37 & {$[22 \sim 24]$} \\
\hline & & $170.00 \sim 313.20$ & $3.110 \sim 69.470$ & $0.00217 \sim 0.02144$ & 23 & {$[21,25,26]$} \\
\hline \multirow{2}{*}{ PS } & \multirow{3}{*}{$\mathrm{N}_{2}$} & $332.20 \sim 333.23$ & $3.050 \sim 50.110$ & $0.00109 \sim 0.02077$ & 21 & {$[21,25]$} \\
\hline & & $352.20 \sim 373.20$ & $2.989 \sim 62.500$ & $0.00106 \sim 0.01813$ & 32 & {$[21,22,25]$} \\
\hline \multirow{6}{*}{ PP } & & $413.20 \sim 453.20$ & $6.452 \sim 18.011$ & $0.00334 \sim 0.00983$ & 13 & {$[22]$} \\
\hline & \multirow{4}{*}{$\mathrm{CO}_{2}$} & $373.15 \sim 373.20$ & $2.930 \sim 12.550$ & $0.02050 \sim 0.10860$ & 21 & {$[6,7]$} \\
\hline & & $393.20 \sim 423.15$ & $4.120 \sim 14.960$ & $0.02260 \sim 0.10050$ & 25 & {$[6,7]$} \\
\hline & & $432.20 \sim 453.20$ & $4.960 \sim 24.910$ & $0.03230 \sim 0.26170$ & 30 & {$[7,24,25]$} \\
\hline & & $464.20 \sim 483.70$ & $4.970 \sim 24.910$ & $0.03020 \sim 0.24290$ & 16 & {$[7,24,25]$} \\
\hline & & $170.00 \sim 483.70$ & $2.068 \sim 69.470$ & & 283 & \\
\hline
\end{tabular}
统计数据如表 2 所示.

表 1 实验数据表

Table 1 Experimental data in this work 
表 2 优化网络结构的统计数据

Table 2 Topology studies for optimal ANN configuration

\begin{tabular}{lllll}
\hline 隐结点数 & AAD & $R^{2}$ & SD & 适应值 \\
\hline 3 & 0.0072 & 0.9053 & 0.0421 & $1.21 \mathrm{E} \sim 04$ \\
4 & 0.0067 & 0.9134 & 0.0419 & $1.10 \mathrm{E} \sim 05$ \\
5 & 0.0064 & 0.9216 & 0.0419 & $1.34 \mathrm{E} \sim 06$ \\
6 & 0.0065 & 0.9117 & 0.0423 & $1.10 \mathrm{E} \sim 06$ \\
7 & 0.0061 & 0.9821 & 0.0302 & $9.99 \mathrm{E} \sim 06$ \\
$\mathbf{8}$ & $\mathbf{0 . 0 0 5 8}$ & $\mathbf{0 . 9 9 1 4}$ & $\mathbf{0 . 0 1 9 8}$ & $\mathbf{6 . 1 1 E} \sim \mathbf{0 7}$ \\
9 & 0.0060 & 0.9842 & 0.0238 & $2.00 \mathrm{E} \sim 06$ \\
10 & 0.0061 & 0.9801 & 0.0419 & $8.99 \mathrm{E} \sim 06$ \\
11 & 0.0063 & 0.9775 & 0.0423 & $9.91 \mathrm{E} \sim 06$ \\
12 & 0.0064 & 0.9647 & 0.0415 & $9.89 \mathrm{E} \sim 05$ \\
13 & 0.0063 & 0.9505 & 0.0425 & $1.16 \mathrm{E} \sim 05$ \\
14 & 0.0072 & 0.9222 & 0.0419 & $8.98 \mathrm{E} \sim 04$ \\
15 & 0.0077 & 0.9157 & 0.042 & $8.15 \mathrm{E} \sim 04$ \\
\hline
\end{tabular}

显然, 选择误差最小和相关系数较高的网络作为最 优的网络结构, 据表 2 所示, 隐层结点数为 8 时网络模 型最优.

\section{4 结果与讨论}

本文建立了基于混沌自适应 PSO-BP 的 3 层结构为 2-8-1 的神经网络模型, 即模型包含 2 个分别代表压力和 温度的输入结点, 8 个隐层结点和 1 个代表溶解量的输出 结点. 首先将建立的模型分别对 $\mathrm{CO}_{2}$ 在 PS、 $\mathrm{N}_{2}$ 在 PS 和 $\mathrm{CO}_{2}$ 在 PP 的三个子库进行训练和验证. 图 1、图 2 和图 3 分别为三个子库中溶解度的预测值和实验值的对比图, 直线表示预测值等于实验值的理想模型, 星号和圆型分 别表示在训练集和验证集中的预测值.

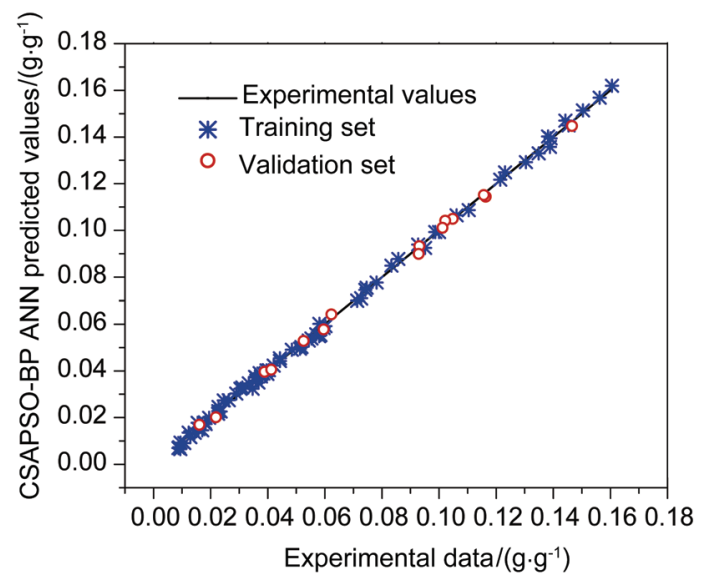

图 1 实验值与模型预测值对比 $\left(\mathrm{PS} / \mathrm{CO}_{2}\right)$

Figure 1 Prediction values vs. experimental data $\left(\mathrm{PS} / \mathrm{CO}_{2}\right)$

在模型的训练和验证集中, 从对比结果可知, 无论 是 $\mathrm{CO}_{2}$ 在 PS、 $\mathrm{N}_{2}$ 在 PS 还是 $\mathrm{CO}_{2}$ 在 PP 集合, 混沌自适 应 PSO-BP 神经网络都能达到很好的模拟效果, 预测值

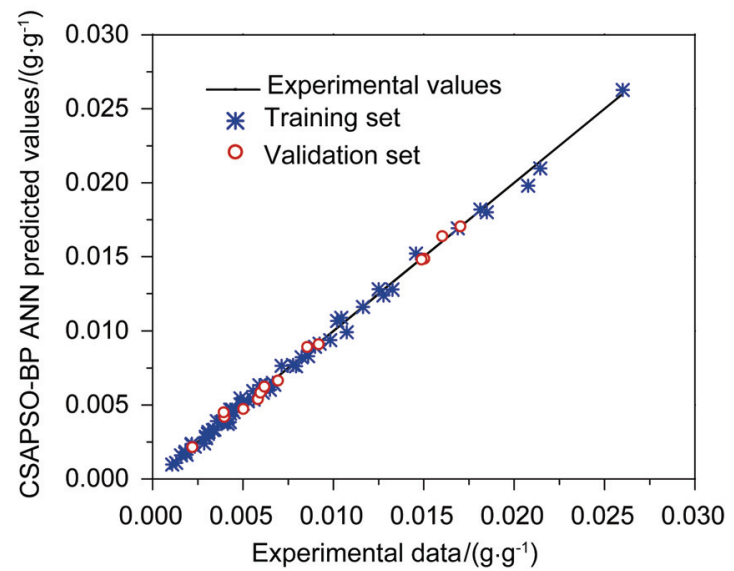

图 2 实验值与模型预测值对比 $\left(\mathrm{PS} / \mathrm{N}_{2}\right)$

Figure 2 Prediction values vs. experimental data $\left(\mathrm{PS} / \mathrm{N}_{2}\right)$

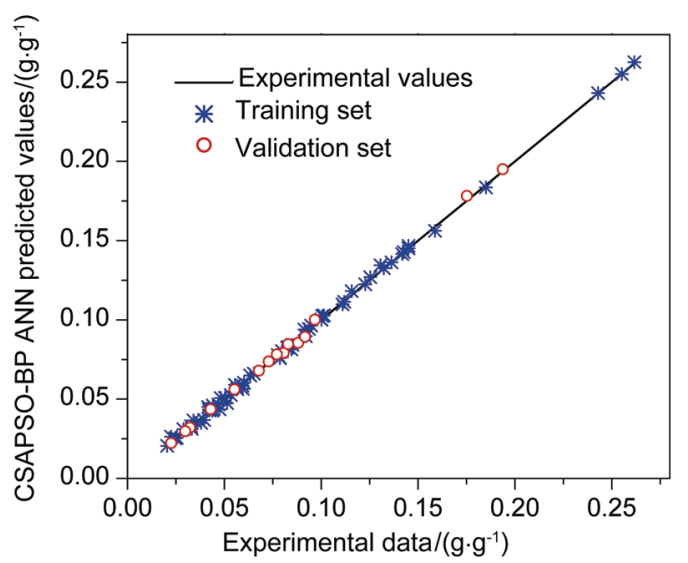

图 3 实验值与模型预测值对比 $\left(\mathrm{PP} / \mathrm{CO}_{2}\right)$

Figure 3 Prediction values vs. experimental data $\left(\mathrm{PP} / \mathrm{CO}_{2}\right)$

与实验值有很好的一致性. 从训练集中的预测结果来 看, 模型已经充分训练; 从验证集中的验证结果来看, 训练好的网络预测具有可靠及高精度的预测能力. 特别 是 $\mathrm{CO}_{2}$ 在 PP 中, 预测相关性更高, 预测值更加吻合实验 值.

利用训练和验证后的混沌自适应 PSO-BP 神经网络 模型进行预测仿真, 将每个集合包含 13 组测试数据的 测试集分别对 $\mathrm{PS} / \mathrm{CO}_{2} 、 \mathrm{PS} / \mathrm{N}_{2}$ 和 $\mathrm{PP} / \mathrm{CO}_{2}$ 进行预测, 图 4 给出了模型预测值与实验值之间的相互关系, 其中曲线 为样本实验值, 星号、加号、圆圈分别表示 $\mathrm{CO}_{2}$ 在 $\mathrm{PP}$, $\mathrm{CO}_{2}$ 在 PS 和 $\mathrm{N}_{2}$ 在 PS 中的模型预测值.

测试结果反映了混沌自适应 PSO-BP 神经网络模型 良好的仿真能力, 三个测试数据库中, 模型的预测值与 实验值都吻合较好. 由图 4 的曲线位置可知, 在一般条 件下, 气体 $\mathrm{N}_{2}$ 在 PS 中的溶解量比 $\mathrm{CO}_{2}$ 小, 而 $\mathrm{CO}_{2}$ 在两 种聚合物的溶解性能相当.

为进一步验证其先进性, 让混沌自适应 PSO-BP 神 经网络与传统 BP 神经网络和 PSO-BP 神经网络的预测 性能进行比较, 比较数据如表 3 所示, 可知, 在相同的 


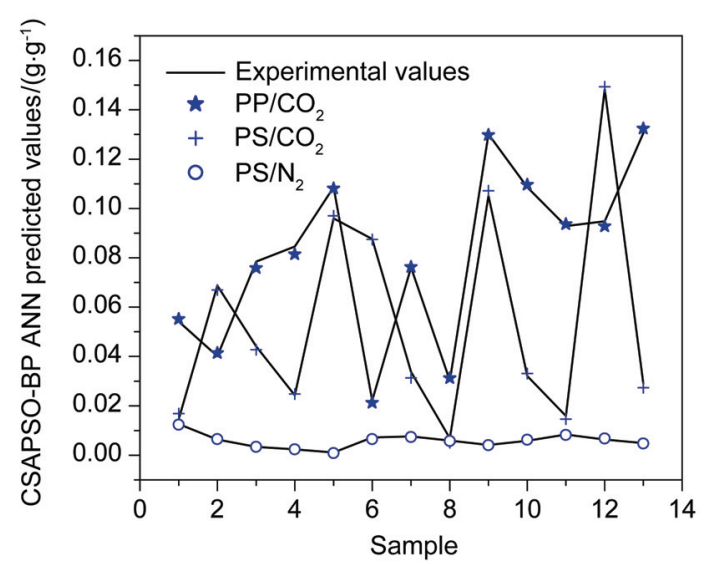

图 4 测试集中实验值与模型预测值的相互关系

Figure 4 Prediction values vs. experimental data in testing set

测试集中, 传统 BP 神经网络、PSO-BP 神经网络和混沌 自适应 PSO-BP 神经网络的预测性能依次上升, 无论是 预测的精度还是相关性混沌自适应 PSO-BP 神经网络模 型均明显较优, 预测值与实验值的吻合度也最好, 预测 能力最强.

上述预测结果从误差和相关性方面表明混沌自适 应 PSO-BP 神经网络的良好预测性能, 分析其优越的原 因, 可归功于其训练算法. 传统的 BP 神经网络采用梯 度下降的 BP 算法训练, 此算法训练时间较长、易陷入 局部极值, 又具有喜新厌旧学习趋势, 从而导致网络不 能完全训练而影响预测效果; 针对 PSO-BP 神经网络, 其训练算法结合了 PSO 算法和 BP 算法各自的优势, 在 很大程度上提高了网络的训练能力, 但也没有解决训练 过程中的早熟问题也使预测性能不太理想; 而混沌自适 应 PSO-BP 神经网络不仅结合了 PSO 算法和 BP 算法各 自的优势, 加入了惯性权重因子, 而且引入的混沌理论 能在训练中早熟问题上进行改进和处理, 给网络的充分 训练提供保证, 从而实现良好的预测性能.

本文提出的预测模型有明显特点, 主要表现在以下 几个方面:

(1) 克服了传统溶解度计算中非稳态 PVT 修正问题.

在传统的溶解性预测模型中, 主要通过气体的溶解 质量和体积两种方式计算溶解度. 在低温低压下, 气体 可以看作为理想气体, 计算结果相对精确. 但在高温高 压下, 如在超临界状态下, 气体不能似为理想气体, 系 统为非稳态, 那么溶解气体的量就得通过 PVT 修正后
计算得到，如通过 SL-EOS 状态方程修正高压膨胀等， 从而影响预测精度. 混沌自适应 PSO-BP 神经网络预测 模型克服了此弊端，从本文得到的结果看，提出的预测 模型从精度和效率上明显优于传统的溶解预测方法.

(2)温度和压力对溶解度的影响.

实验研究表明, 在等压条件下随着温度的增加, 气 体在聚合物中的溶解度呈先下降后上升的趋势 ${ }^{[6,24]}$. 主 要表现两方面的原因: 一方面是温度对气体密度的影 响. 随着温度的升高, 气体的密度下降, 从而溶剂化效 应下降，导致气体在聚合物中的溶解度下降. 另一方面 是温度对系统蒸汽压的影响. 随着温度的升高, 系统的 蒸汽压增大, 导致气体在聚合物中的溶解度增大. 这两 个相反的影响结果导致溶解度呈先下降后上升的趋势, 并出现溶解度最低点. 研究表明, 在溶解度最低点温度 以下, 温度对密度的影响占主导地位使溶解度呈下降趋 势; 在溶解度最低点温度以上, 温度对蒸汽压的影响占 主导使溶解度呈上升趋势. 另外, 在温度一定的条件下， 随着压力的增加, 气体在聚合物中的溶解度一般都呈上 升的趋势.

温度和压力对溶解度的影响表现出非常复杂的非 线性关系, 而混沌自适应 PSO-BP 神经网络能根据温度 和压力对溶解度的影响, 利用人工神经网络黑盒子模型 具有较强的模拟非线性映射的特点，模拟得到与实验现 象完全吻合的溶解规律，提供一种确实可行的溶解度预 测与分析方法.

(3)不受高温高压等约束条件的限制.

传统的溶解计算是在某些假设情形中计算, 如在温 度不高和压力不太大时假设气体为理想气体等, 过多的 系统假设将背离真实的实验情况, 势必影响预测精度. 而混沌自适应 PSO-BP 神经网络溶解模型不受很多约束 条件的限制.

(4)模型的扩展.

从气体在聚合物中的溶解行为分析可知, 影响溶解 性的因素很多, 除了温度和压力外, 还和聚合物与溶剂 的极性、结晶程度与晶区大小和溶解度本身的参数等有 关. 虽然, 针对特定的溶剂与溶质, 温度和压力为影响 溶解性的主要因素, 但为提供更完善的预测模型, 其他 影响因素将在今后的研究中完善. 本文对两种气体在两 种聚合物中的溶解性进行了研究, 在今后的工作中也将 继续扩展研究对象，使提出的模型更具有综合性.

表 3 各预测模型测试结果比较

Table 3 Statistical comparison results in this work

\begin{tabular}{|c|c|c|c|c|c|c|c|c|c|}
\hline \multirow{2}{*}{ 测试物 } & \multicolumn{3}{|c|}{ 传统 BP 神经网络 } & \multicolumn{3}{|c|}{ PSO-BP 神经网络 } & \multicolumn{3}{|c|}{ 混沌自适应 PSO-BP 神经网络 } \\
\hline & $\mathrm{AAD}$ & $R^{2}$ & SD & $\mathrm{AAD}$ & $R^{2}$ & SD & $\mathrm{AAD}$ & $R^{2}$ & SD \\
\hline $\mathrm{PS} / \mathrm{CO}_{2}$ & 0.0703 & 0.9502 & 0.0976 & 0.0131 & 0.9723 & 0.0684 & 0.0057 & 0.9914 & 0.0201 \\
\hline $\mathrm{PS} / \mathrm{N}_{2}$ & 0.0811 & 0.9484 & 0.0998 & 0.0102 & 0.9702 & 0.0701 & 0.0061 & 0.9907 & 0.0213 \\
\hline $\mathrm{PP} / \mathrm{CO}_{2}$ & 0.0678 & 0.9601 & 0.0945 & 0.0125 & 0.9736 & 0.0637 & 0.0055 & 0.9921 & 0.0180 \\
\hline 平均值 & 0.0731 & 0.9529 & 0.0973 & 0.0119 & 0.9720 & 0.0674 & 0.0058 & 0.9914 & 0.0198 \\
\hline
\end{tabular}


在模型应用前景的推广上, 可供许多复杂的预测领 域提供一种新的方法和实现思路, 特别是在化工领域 中, 如在设计中分析材料组分、工艺和性能之间的规律, 在加工中高温变形和流变应力的分析, 在工艺参数的优 化研究, 力学性能研究及在材料热处理等领域都有很好 的应用前景.

\section{5 结论}

本文构建了基于混沌自适应 PSO-BP 神经网络的气 体在聚合物中的溶解预测模型, 预测结果能很好的与实 验结果吻合, 达到了非常满意的预测效果. 通过预测实 例表明混沌自适应 PSO-BP 神经网络进行溶解性预测的 可行性与准确性, 预测能力很强, 操作也简便, 预测成 本低等优点. 作者也将在今后的研究工作中继续关注此 学科的研究发展, 并加强理论学习开拓更广的相关研究 领域.

\section{References}

[1] Merker, T.; Vrabec, J.; Hasse, H. Fluid Phase Equilib. 2012, 315, 77.

[2] Wang, J.; Zhu, W. L.; Zhang, H. T.; Park, C. B. Chem. Eng. Sci. 2012, 75, 390 .

[3] Liu, S.; Zhang, J.; Zhang, Y.; Qin, L. Acta Chim. Sinica 2012, 70, 1511. (刘树深, 张瑾, 张亚辉, 覃礼堂, 化学学报, 2012, 70, 1511.)

[4] Yu, X. Acta Chim. Sinica 2010, 68, 2264. (禹新良, 化学学报, 2010, 68, 2264.)

[5] Nalawade, S. P.; Picchioni, F.; Janssen, L. Prog. Polym. Sci. 2006, $31,19$.

[6] Li, D. C.; Liu, T.; Zhao, L.; Yuan, W. K. Ind. Eng. Chem. Res. 2009, $48,7117$.
[7] Lei, Z. G.; Ohyabu, H.; Sato, Y.; Inomata, H.; Smith, R. L. J. Supercrit. Fluids 2007, 40, 452.

[8] Bian, X.; Du, Z.; Tang, Y. Petrochem. Technol. 2011, 40, 536. (六小 强, 杜志敏, 汤勇, 石油化工, 2011, 40, 536.

[9] Zhang, Q.; Feng, X.; Long, H.; Suo, J.; Zhang, D.; Xu, L.; Xu, L. Acta Chim. Sinica 2012, 70, 989. (张庆友, 冯秀林, 龙海林, 索净 洁, 张丹丹, 许力壮, 许禄, 化学学报, 2012, 70, 989.)

[10] Gharagheizi, F.; Eslamimanesh, A.; Mohammadi, A. H.; Richon, D. Ind. Eng. Chem. Res. 2011, 50, 221

[11] Ma, Y.; Shi, W.; Zhao, C.; Yang, D.; Lu, Q.; Li, S.; Tu, J.; Wang, W.; Fan, Z. Acta Chim. Sinica 2011, 69, 719. (马艳, 石文鹏, 赵辰 阳, 杨冬梅, 逯琪, 李速明, 涂建军, 王巍, 范仲勇, 化学学报, 2011, 69, 719.)

[12] Yu, D.; Zhou, G.; Ji, F.; Li, S.; Yang, D.; Wang, T.; Cao, L. Acta Chim. Sinica 2011，69，960. (虞丹尼，周光明，吉芳英，黎司，杨 大成, 王图锦, 曹琳, 化学学报, 2011, 69, 960.)

[13] Bakhbakhi, Y. Math. Comput. Model. 2012, 55, 1932.

[14] Lashkarbolooki, M.; Vaferi, B.; Rahimpour, M. R. Fluid Phase Equilib. 2011, 308, 35.

[15] Eslamimanesh, A.; Gharagheizi, F.; Mohammadi, A. H.; Richon, D. Chem. Eng. Sci. 2011, 66, 3039.

[16] Yun, T.; Junsong, L. U. O. Comput. Simul. 2011, 28, 219.

[17] Ding, H.; Wu, J.; Li, X. In Advances in Swarm Intelligence. Third International Conference, Eds.: Tan, Y.; Shi, J.; Ji, Z., SpringerVerlag, Shenzhen, 2012, pp. 444 451.

[18] Ahmadi, M. A. Fluid Phase Equilib. 2012, 314, 46

[19] Zhang, J. R.; Zhang, J.; Lok, T. M.; Lyu, M. R. Appl. Math. Comput. 2007, 185, 1026.

[20] Sun, Y.; Wang, Z.; Qi, G.; Van Wyk, B. J. Eng. Optimiz. 2011, 43, 19.

[21] Hilic, S.; Boyer, S.; Padua, A.; Grolier, J. J. Polym. Sci. B-Polym. Phys. 2001, 39, 2063.

[22] Sato, Y.; Yurugi, M.; Fujiwara, K.; Takishima, S.; Masuoka, H. Fluid Phase Equilib. 1996, 125, 129.

[23] Sato, Y.; Takikawa, T.; Takishima, S.; Masuoka, H. J. Supercrit. Fluids 2001, 19, 187.

[24] Khajeh, A.; Modarress, H.; Mohsen-Nia, M. Iran. Polym. J. 2007, 16,759 .

[25] Sato, Y.; Fujiwara, K.; Takikawa, T.; Sumarno; Takishima, S.; Masuoka, H. Fluid Phase Equilib. 1999, 162, 261.

[26] Newitt, D. M.; Weale, K. E. J. Chem. Soc. 1948, 1039, 1541 